Portland State University

PDXScholar

Electrical and Computer Engineering Faculty

Publications and Presentations

$5-1-2007$

\title{
Non-Laser-Based Scanner for Three-Dimensional Digitization of Historical Artifacts
}

\author{
Donald D. Duncan \\ Portland State University \\ Daniel V. Hahn \\ Johns Hopkins University \\ Kevin C. Baldwin \\ Johns Hopkins University
}

Follow this and additional works at: https://pdxscholar.library.pdx.edu/ece_fac

Part of the Electrical and Computer Engineering Commons

Let us know how access to this document benefits you.

Citation Details

D. V. Hahn, K. C. Baldwin, D. D. Duncan, "Non-laser based scanner for 3D digitization of historical artifacts," Applied Optics, V. 46, pp. 2838-2850 (2007)

This Article is brought to you for free and open access. It has been accepted for inclusion in Electrical and Computer Engineering Faculty Publications and Presentations by an authorized administrator of PDXScholar. Please contact us if we can make this document more accessible: pdxscholar@pdx.edu. 


\title{
Non-laser-based scanner for three-dimensional digitization of historical artifacts
}

\author{
Daniel V. Hahn, Kevin C. Baldwin, and Donald D. Duncan
}

\begin{abstract}
A 3D scanner, based on incoherent illumination techniques, and associated data-processing algorithms are presented that can be used to scan objects at lateral resolutions ranging from 5 to $100 \mu \mathrm{m}$ (or more) and depth resolutions of approximately $2 \mu \mathrm{m}$. The scanner was designed with the specific intent to scan cuneiform tablets but can be utilized for other applications. Photometric stereo techniques are used to obtain both a surface normal map and a parameterized model of the object's bidirectional reflectance distribution function. The normal map is combined with height information, gathered by structured light techniques, to form a consistent 3D surface. Data from Lambertian and specularly diffuse spherical objects are presented and used to quantify the accuracy of the techniques. Scans of a cuneiform tablet are also presented. All presented data are at a lateral resolution of $26.8 \mu \mathrm{m}$ as this is approximately the minimum resolution deemed necessary to accurately represent cuneiform. () 2007 Optical Society of America

OCIS codes: $150.6910,100.2000,100.5070,110.6880,120.2830,120.6650$.
\end{abstract}

\section{Introduction}

In recent years there has been a push to develop a means of digitizing cuneiform tablets, the world's oldest known writing system. ${ }^{1,2}$ Unlike most other forms of documentation, cuneiform is $3 \mathrm{D}$ in nature. The media are rounded clay tablets (with writing often wrapping around the sides of the tablet), and the characters are wedge impressions made when the clay was moist (Figure 7 below shows a photograph of a cuneiform tablet). The quest for digitization is fueled by several factors. Only a small fraction of the estimated 500,000 tablets known to exist can be made available for public display. ${ }^{3}$ Even for cuneiform scholars, of which there are few, access to worldwide collections is expensive, time-consuming, and can be politically difficult. ${ }^{1,4}$

The Cuneiform Digital Library Initiative, ${ }^{5}$ an international collaboration among many universities and museums, has utilized a flatbed scanner to aid

The authors are with The Johns Hopkins University Applied Physics Laboratory, 11100 Johns Hopkins Road, Laurel, Maryland, 20723-6099, USA. D. Hahn's e-mail address is daniel.hahn@ jhuapl.edu.

Received 3 October 2006; revised 2 January 2007; accepted 24 January 2007; posted 24 January 2007 (Doc. ID 75751); published 1 May 2007.

0003-6935/07/152838-13\$15.00/0

(C) 2007 Optical Society of America in abstracting pictographic symbols from cuneiform tablets. ${ }^{2}$ This approach, along with other $2 \mathrm{D}$ means of preservation, falls short of preserving much of the information resident on cuneiform tablets, such as the tablet material properties, writing that wraps around the tablet, and author-dependent features including wedge depth or accent, and character size. ${ }^{6}$

Several researchers have utilized laser-based scanners to obtain a 3D digitization of cuneiform tablets. ${ }^{2,3}$ While superior in data content to $2 \mathrm{D}$ methods, laser scanners have their own shortcomings. Laser systems are expensive ${ }^{3}$ and subject to error attributable to speckle noise and multiple surface reflections in deep grooves. ${ }^{4}$ In addition, there is an inherent trade-off between the depth of field and resolution of the scan. ${ }^{4}$ Both Kumar et al. ${ }^{1}$ and Woolley et al. ${ }^{3}$ cite $50 \mu \mathrm{m}$ as the best attainable lateral resolution for a laserbased system; this is also the resolution achieved by Anderson and Levoy. ${ }^{2}$ Several scholars have indicated that $50 \mu \mathrm{m}$ resolution is insufficient and argue that a lateral resolution of at least $25 \mu \mathrm{m}$ is required. ${ }^{1,3,6}$

Holographic recording schemes have also been used to obtain shape data on cuneiform tablets 7,8 ; Dreesen et al. ${ }^{8}$ were able to obtain a lateral resolution of $3 \mu \mathrm{m}$. However, this scheme still requires multiple holograms to capture all faces of the object, and the resulting holograms would quickly prove cumbersome for the sharing and storage of information. Most 
importantly, holograms are an analog storage medium. To alleviate the problem of data storage and sharing, as well as to aid in visualization and any subsequent processing, the digitization of the surface information is of paramount importance. Digitization using a holographic technique would require recording of the interference pattern via a CCD. ${ }^{9}$ In addition, computer intensive numerical reconstructions would be required to visualize the object.

We present a 3D scanner, based on incoherent illumination, which is designed to digitize cuneiform tablets at lateral resolutions as small as $5 \mu \mathrm{m}$ and depth resolution of approximately $2 \mu \mathrm{m}$. The scanner utilizes a camera and telecentric lens to acquire images of tablets under varying controlled illumination conditions. Image data are processed using photometric stereo and structured light techniques to determine the tablet shape and surface reflectance characteristics, namely the bidirectional reflectance distribution function (BRDF). Color information is reconstructed from primary color monochrome image data. A preliminary version of the scanner and associated data processing algorithms has been previously detailed as a work in progress, ${ }^{4}$ but was lacking in several key regards. First, a Lambertian BRDF was assumed; we now discuss a means by which to solve for the actual BRDF of the tablet material, a feature that is important for proper shape reconstruction and material identification. Second, we present scans of test objects that validate the data and bound the measurement error. In addition, several algorithmic improvements are detailed.

\section{Scanner Design and Operation}

A diagram of the scanner is shown in Fig. 1. The scanner uses fixed camera and object positions to maintain image registration while varying the lighting conditions by rotating the source about the object and by projecting different illumination colors and patterns (the polar angle of the source is fixed). A digital projector was selected as the light source as it provides excellent illumination uniformity, can easily project custom patterns, and, through the use of an additional lens, provides adequate collimation. The object is imaged with a telecentric lens; it is the magnification factor of this lens and the pixel size of the camera that determine the horizontal resolution of the scanner (current camera technology limits this resolution to approximately $5 \mu \mathrm{m}$ when using a telecentric lens of unity magnification). Sharp image focus is obtained by attaching a neutral density (ND) filter to the telecentric lens so that the iris of the lens is fully opened. A V block mounted on top of an elevation stage is used to position the object within the working distance of the telecentric lens.

Scanner usage and associated data processing steps are detailed in the flow chart shown in Fig. 2. Color information is obtained by illuminating the object with solid primary colors over various azimuthal angles. Shape information is obtained by combining two techniques: the method of photometric stereo, ${ }^{10}$ which provides information that is locally accurate, and structured light analysis, ${ }^{11,12}$ which provides global accuracy. Photometric stereo methods are also

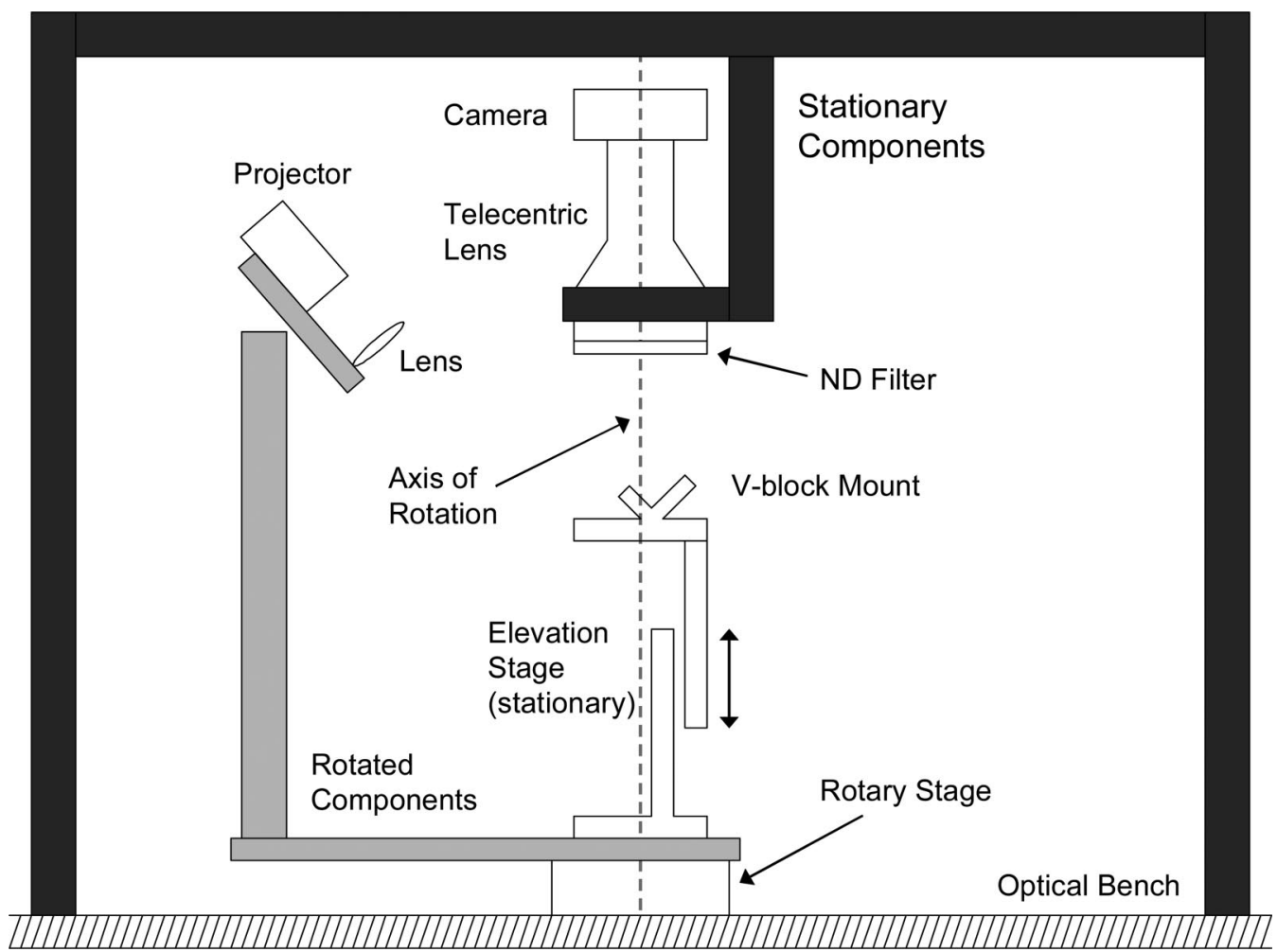

Fig. 1. Scanner design (Ref. 4). 


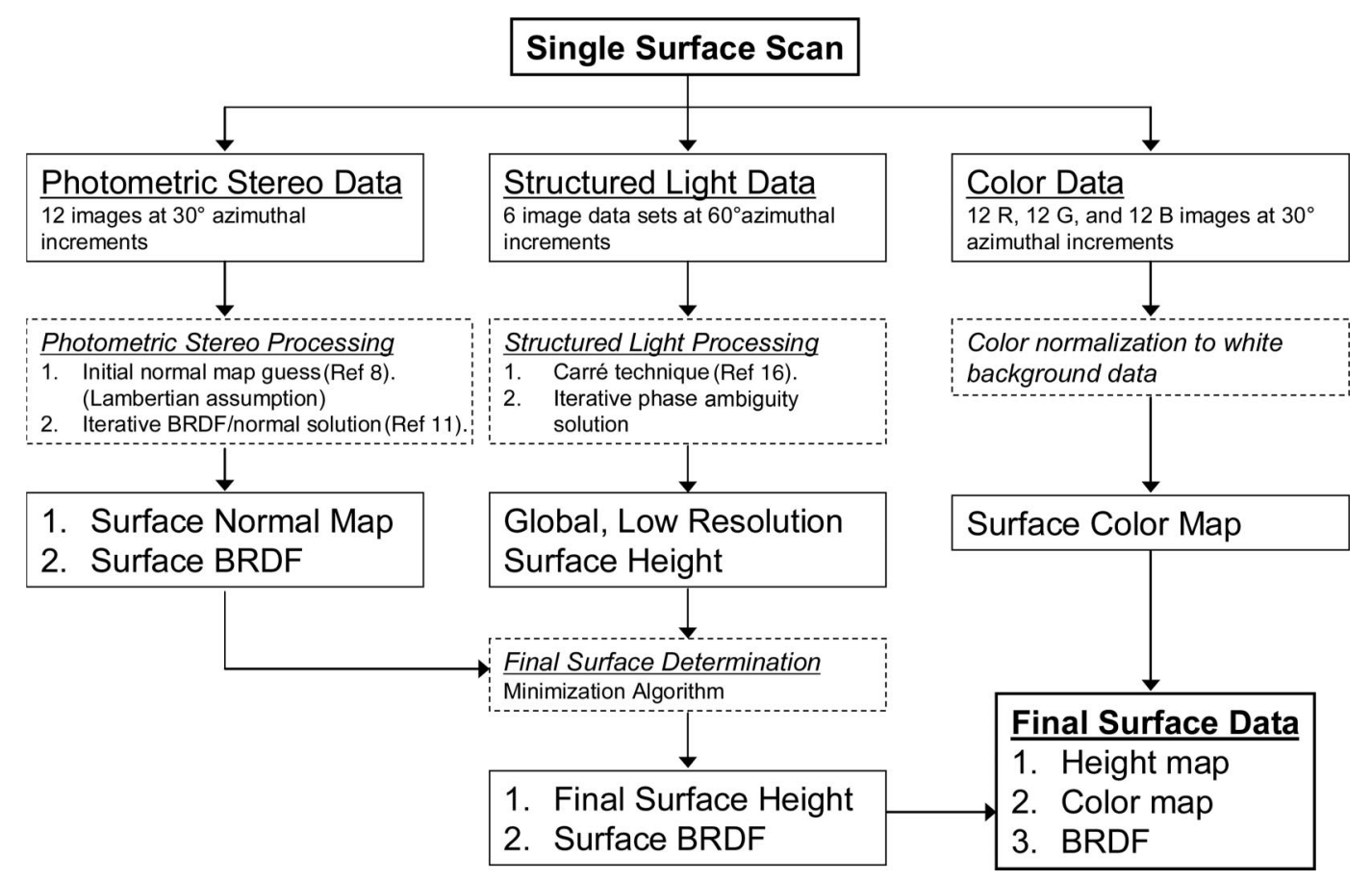

Fig. 2. Scanner operation.

used to recover the surface BRDF. ${ }^{13}$ Each image is processed, upon acquisition, to correct for camera noise, camera nonlinearity, and illumination nonuniformity. ${ }^{14}$

\section{A. Application of Photometric Stereo}

The method of photometric stereo is used to obtain a surface normal map of the object and to determine the surface BRDF in accordance with a parameterized model, specifically the Ward model. ${ }^{15}$ This is accomplished by acquiring images over various azimuthal angles under a collimated white-light source. The brightness of each pixel in each image is dependent upon the illumination, view, and normal directions, as well as the BRDF of the surface. Given the data and the known illumination and view directions, the BRDF and normal map are iteratively estimated. The normal data resulting from photometric stereo analysis can be integrated over small areas to obtain good estimates of the surface height. Unfortunately, the normal map does not form a conservative surface, and small errors accumulate when integration is attempted over larger areas; the data are locally accurate but suffer larger scale inaccuracies. ${ }^{16,17}$

\section{B. Application of Structured Light}

The structured light technique implemented projects a series of $1 \mathrm{D}$ sinusoidal patterns onto the object at a fixed polar and various azimuthal angles. By acquiring data at multiple azimuthal projection angles, it is possible to determine surface orientations that may be under shadow at a subset of projection angles. However, there is still a limit to the steepness of surfaces that can be measured; the limitations to structured light measurement involving phase-to-height conversion algorithms are quantified by Zhang and Huang. ${ }^{18}$

At each projection angle, four patterns, each out of phase with one another by $90^{\circ}$, are projected for each of a series of iteratively doubled frequencies. Each of the images of different phase for a single frequency is used to determine a phase value that is unaffected by variations in surface reflectance. This processing is performed using the Carré technique of phasemeasurement interferometry. ${ }^{19}$ The resulting images are compared to images of a flat white background to calculate the phase difference and corresponding relative object height. ${ }^{12}$ The resulting height data, although sampled at the same resolution as the normal data, are inherently lower in resolution. This results in the opposite characteristics of the surface normal data-globally accurate but of low resolution.

\section{Combination of Normal and Height Data}

The two preceding analysis techniques form a synergistic data set that contains all information necessary to construct an accurate surface map of the object. The global, low resolution height from structured light analysis is combined with the locally accurate normal data from photometric stereo via an error minimization algorithm; this results in a high resolution data set that is geometrically accurate on both the global and local scales.

\section{Processing Algorithms}

\section{A. Photometric Stereo}

The technique of photometric stereo is used to calculate both the surface normal map and the BRDF of 
the object. The main premise of the technique is that a surface will appear brighter when the illumination direction converges toward either the surface normal (for a Lambertian reflector) or the angle of specular reflection with respect to the camera (for a diffusely specular surface). By acquiring enough images under controlled illumination conditions, it is possible to both reconstruct the BRDF of the object surface and to determine the surface normal map. In principle, three images are the minimum required for calculation of the surface normal map. ${ }^{10}$ To reconstruct the $\mathrm{BRDF}$, one additional image is required for each parameter of the BRDF model. However, it is beneficial to acquire more images than are absolutely necessary so that the error attributable to measurement noise is reduced. Acquiring additional images also mitigates the shadowing problems.

The photometric stereo algorithm implemented is similar to that of Goldman et al.,13 but has several key differences. In particular, rather than allowing the BRDF to vary for each individual pixel, we assume that it is constant over the object surface. In addition, we do not assume known material types or characteristics and fit the parameters of the Ward model $^{15}$ directly (instead of adjusting the material weights for preset Ward parameters).

The initial step of the algorithm assumes a Lambertian BRDF and solves for the normal map via the method detailed by Woodham. ${ }^{10}$ The intensity values of the point $(x, y)$ for a series of images acquired under uniform and collimated illumination are written as

$$
\bar{I}=Q \overline{\bar{N}} \bar{n},
$$

where $Q$ is the reflectance of the point, $\overline{\bar{N}}$ is a matrix, which describes the directions of incident illumination, and $\bar{n}$ is the surface normal at $(x, y)$. Although only three images are required to uniquely invert Eq. (1), more are used and a least-squares approach is taken to reduce error and to account for shadowed facets. Defining the $z$ axis to point downward from the camera toward the object, Eq. (1) becomes

$$
\begin{aligned}
{\left[\begin{array}{c}
I_{1} \\
\vdots \\
I_{K}
\end{array}\right]=} & Q\left[\begin{array}{ccc}
\sin (\theta) \sin \left(\phi_{1}\right) & \sin (\theta) \cos \left(\phi_{1}\right) & -\cos (\theta) \\
\vdots & \vdots & \vdots \\
\sin (\theta) \sin \left(\phi_{K}\right) & \sin (\theta) \cos \left(\phi_{K}\right) & -\cos (\theta)
\end{array}\right] \\
& \times\left[\begin{array}{l}
n_{x} \\
n_{y} \\
n_{z}
\end{array}\right],
\end{aligned}
$$

where $\theta$ is the polar angle and $\phi$ is the azimuthal angle. The least-squares solution is

$$
Q \bar{n}=\left[\left(\overline{\bar{N}}^{T} \overline{\bar{N}}\right)^{-1} \overline{\bar{N}}^{T}\right] \bar{I} .
$$

The normal map resulting from Eq. (3) is used as an initial guess in an iterative algorithm that first optimizes the parameters of the BRDF and then updates the normal map. A three-parameter Ward
BRDF model is used ${ }^{15}$ :

$\rho\left(\theta_{i}, \theta_{r}\right)=\frac{\rho_{L}}{\pi}+\frac{\rho_{s}}{\sqrt{\cos \left(\theta_{i}\right) \cos \left(\theta_{r}\right)}} \frac{\exp \left(-\tan ^{2}(\delta) / \beta^{2}\right)}{4 \pi \beta^{2}}$,

where the independent parameters are the coefficient of the Lambertian term, $\rho_{L}$, the coefficient of the specular term, $\rho_{s}$, and the width of the specular term, $\beta$. In Eq. (4), $\theta_{i}, \theta_{r}$, and $\delta$ are the angles between the normal and the illumination directions, $\bar{L}$, view direction, $\bar{V}$, and halfway vector, $\bar{h}=(\bar{L}+\bar{V}) /\|\bar{L}+\bar{V}\|$, respectively. Parameter optimization is performed using the Levenberg-Marquardt nonlinear optimization algorithm ${ }^{20}$ with the objective function

$$
\psi=\sum_{k, p}\left[I_{k, p}-f\left(\overline{n_{p}}, \overline{L_{k}}, \rho_{L}, \rho_{s}, \beta\right)\right]^{2},
$$

where $I_{k, p}$ represents the observed intensity of each pixel $p$ in each image $k$, and $f\left(\overline{n_{p}}, \overline{L_{k}}, \rho_{L}, \rho_{s}, \beta\right)$ is the theoretical intensity according to the pixel normal, illumination direction, and BRDF model.

Once the BRDF parameters have been determined, an updated normal map is calculated. Unfortunately, this is not trivial for the case of a non-Lambertian $\mathrm{BRDF}$; the only way to perform this operation is to minimize the objective function, $\psi$, with respect to the surface normals. ${ }^{13}$ In other words, a search is performed over the upward facing hemisphere by varying the normals and keeping the Ward parameters constant. The exit criterion for this condition is a change in all three Ward parameters of less than $0.1 \%$.

As previously noted, the normal map resulting from this approach does not form a conservative surface owing to the nature of the point-by-point calculations. The integration from the normal map to a height field is path dependent and results in unrealistic shapes when performed on a global scale. To counter these problems, structured light data are incorporated into the final surface determination.

\section{B. Structured Light}

The basic premise of the structured light technique employed is to measure the phase shift of a sinusoidal pattern projected onto the object versus onto a flat background. The resulting phase difference is proportional to the relative object height where the constant of proportionality is determined by applying the technique to a flat object of known height. ${ }^{12}$ There are three main problems with this approach. First, each projection angle results in some of the object features being shadowed. This problem is resolved by using multiple projection angles and statistical analysis to intelligently select an appropriate final value of the phase difference at the point $(x, y)$. If a final value at a given point cannot be determined within an acceptable degree of certainty, the hole is filled when the data are combined with the normal map to construct the final surface. Such cases might arise due to mea- 
surement error on a multifaceted area imaged by a single pixel or due to the lack of enough independent phase measurements (from different azimuthal projection angles) in areas of deep grooves consistently under shadow or areas of steep slope with respect to the view direction.

The second problem with this approach is that illumination nonuniformities, camera noise, and variations in surface reflectance and orientation make it difficult to accurately measure phase. This is especially true when viewing a textured object such as a cuneiform tablet. Various techniques of phasemeasurement interferometry can be used to solve this problem as they are not dependent upon local reflectance or illumination level. We have chosen to implement the Carré technique, which requires that four images of differing phase (but the same frequency and amplitude) be acquired. A value of the phase, $\varphi$, at each projection frequency is then calculated via the relation ${ }^{19}$

$$
\varphi=\tan ^{-1}\left[\frac{\sqrt{\left(I_{1}-I_{4}+I_{2}-I_{3}\right)\left[3\left(I_{2}-I_{3}\right)-\left(I_{1}-I_{4}\right)\right]}}{I_{2}+I_{3}-I_{1}-I_{4}}\right],
$$

where the $I_{x}$ represent four 1D sinusoidal patterns, each out of phase with its predecessor by a constant phase shift. The quadrants of $\varphi$ are determined by examination of the signs of quantities proportional to $\sin (\varphi)$ and $\cos (\varphi)^{21}$ :

$$
\begin{array}{r}
I_{2}-I_{3} \propto \sin (\varphi), \\
I_{2}+I_{3}-I_{1}-I_{4} \propto \cos (\varphi) .
\end{array}
$$

Although only three images are needed to uniquely solve for the phase, the Carré technique uses four, and thereby reduces error due to measurement uncertainty. It also has the advantage of working when a linear phase shift is introduced in a converging or diverging beam where the amount of phase shift varies across the beam. ${ }^{19}$ In other words, the experiment will still work even if the projection beam is not perfectly collimated.

The third and final problem associated with the implemented structured light technique involves the trade-off between the measurement error and phase ambiguity. In short, the greater the period of the sinusoid, the greater the measurement error. However, short period sinusoids lead to an increase in phase difference ambiguities $( \pm n 2 \pi)$. An iterative approach is taken in which the frequency of the projected sinusoids are doubled, and the resulting value of $\varphi$ is used to refine the original value of $\varphi$. Thus the highest resolution sinusoid is used to determine the phase, and the iteratively frequency-halved sinusoids are used to resolve the $\pm n 2 \pi$ ambiguities.

The shortcoming of this particular implementation of the structured light technique is that it is intrin- sically low in resolution, both laterally and axially. The projector is lower in resolution (number of pixels) than the camera and also overfills the area viewed by the camera. In addition, the acquired images are subject to intrinsic camera noise; this contributes to uncertainty in high resolution phase values. The end result is an oversampled and low resolution surface, in comparison to the normal map obtained using the method of photometric stereo. However, the benefit of this structured light technique is its high level of global accuracy, which is unattainable by the photometric stereo method.

\section{Final Surface Determination}

The photometric stereo and structured light results are complementary data sets that contain the information necessary to construct an accurate surface map of an object. The normal map resulting from photometric stereo analysis does not form a conservative surface, and integration of the data yields global shape inaccuracies. The resolution of the normal data, however, is excellent. Structured light measurements, on the other hand, provide globally accurate height information that is inherently consistent but low in resolution. An iterative minimization algorithm was therefore designed to combine the data sets in such a way as to take advantage of the benefits of each and to mitigate the drawbacks.

Two main constraints are incorporated into the algorithm. The first minimizes the error between the slope of the final surface and the normal map on a point-by-point basis, ${ }^{22}$ thereby taking advantage of the high resolution of the normal data and avoiding problems due to large-path integration. The second constraint minimizes the relative height difference between the final surface and a median filtered structured light height map. This constraint uses the global accuracy of the height data while removing the effects attributable to isolated noisy data points. A complete description of the algorithm follows.

The height of the object surface is updated according to the rule

$$
\begin{aligned}
h_{n+1}(x, y)= & h_{n}(x, y)+\left\{[1-\lambda(x, y)] \delta h_{P}(x, y)\right. \\
& \left.+\lambda(x, y) \delta h_{S L}(x, y)\right\} .
\end{aligned}
$$

In this equation, $\delta h_{S L}$ is the difference between the median filtered height, $h_{S L}$, and the surface height

$$
\delta h_{S L}(x, y)=h_{S L}(x, y)-h_{n}(x, y) \text {, }
$$

$\lambda$ is a weighting factor bound to the interval $[0,0.5]$,

$$
\lambda(x, y)= \begin{cases}\left(\delta h_{S L}(x, y) / \chi\right)^{2} / 2 ; & \delta h_{S L}(x, y)<\chi \\ 1 / 2 ; & \text { otherwise }\end{cases}
$$

$\chi$ is the lateral image resolution (product of the pixel size and telecentric lens magnification); and $\delta h_{P}$ is the 
height error calculated by comparing the shape of the current surface to the normal data

$$
\begin{aligned}
\delta h_{P}(x, y)= & \frac{\chi}{4}\left[\delta S_{x}(x-1, y)-\delta S_{x}(x+1, y)\right. \\
& \left.+\delta S_{y}(x, y-1)-\delta S_{y}(x, y+1)\right],
\end{aligned}
$$

where $\delta \bar{S}$ is the slope error

$$
\delta \bar{S}(x, y)=\bar{S}(x, y)-\bar{S}_{P}(x, y) .
$$

$\bar{S}$ is the slope as calculated from the surface height,

$$
\begin{aligned}
& S_{x}(x, y)=\frac{h_{n}(x-1, y)-h_{n}(x+1, y)}{2 \chi} ; \\
& S_{y}(x, y)=\frac{h_{n}(x, y-1)-h_{n}(x, y+1)}{2 \chi},
\end{aligned}
$$

and $\bar{S}_{P}$ is the slope measured by photometric stereo analysis

$$
\bar{S}_{P}(x, y)=-\frac{n_{x}(x, y)}{n_{z}(x, y)} \hat{x}-\frac{n_{y}(x, y)}{n_{z}(x, y)} \hat{y} .
$$

The initial guess, $h_{0}$, used in the algorithm is a $4 \times 4$ block-integrated surface (the $x$ - and $y$-slope maps are combined and locally integrated using the Fried algorithm ${ }^{23}$ ) where the shape of each block is determined by integration of the normal data. The center height of each block is set to the average height over the region as measured by structured light analysis. An average height adjustment of less than $\chi / 100$ is used as the exit criterion for the algorithm, with the added restraint that at least ten iterations be performed.

\section{Color Map}

The color map of the object, $\bar{C}$, is calculated by multiplying the reflectance data $(Q)$ determined from photometric stereo analysis by a normalized red-green-blue (RGB) color map of the object. This normalized color map is obtained by summing and normalizing the background corrected monochrome images (red, green, and blue) over all azimuthal angles of data acquisition. Specifically,

$\bar{C}=Q \times \frac{\left[\begin{array}{lll}C_{R} & C_{G} & C_{B}\end{array}\right]}{\left|\left[\begin{array}{lll}C_{R} & C_{G} & C_{B}\end{array}\right]\right|}, \quad$ where

$C_{X}=\sum_{\phi}$ (background corrected $\mathrm{r}, \mathrm{g}$, or b color images).

\section{Results}

All data presented hereafter have been acquired using a camera with square pixels $6.7 \mu \mathrm{m}$ in size and a telecentric lens of $4 \times$ magnification, making each data point $26.8 \mu \mathrm{m}$ apart laterally. The finest reso- lutions of structured light phase images were projections of each sinusoidal cycle over a lateral distance of approximately $0.6 \mathrm{~mm}$. We present results from two spherical test objects, as well as an actual cuneiform tablet (denoted T24).

Note that lateral resolutions as low as $5 \mu \mathrm{m}$ can be realized by replacing the imaging camera with one having $5 \mu \mathrm{m}$ square pixels and the imaging lens with one of unity $(1 \times)$ magnification. However, these changes would also reduce the field of view of the scanner by a factor of 4 in both lateral dimensions. The imaging components used were chosen to best optimize this trade-off between the lateral resolution and the scanner field of view for the purpose of scanning cuneiform.

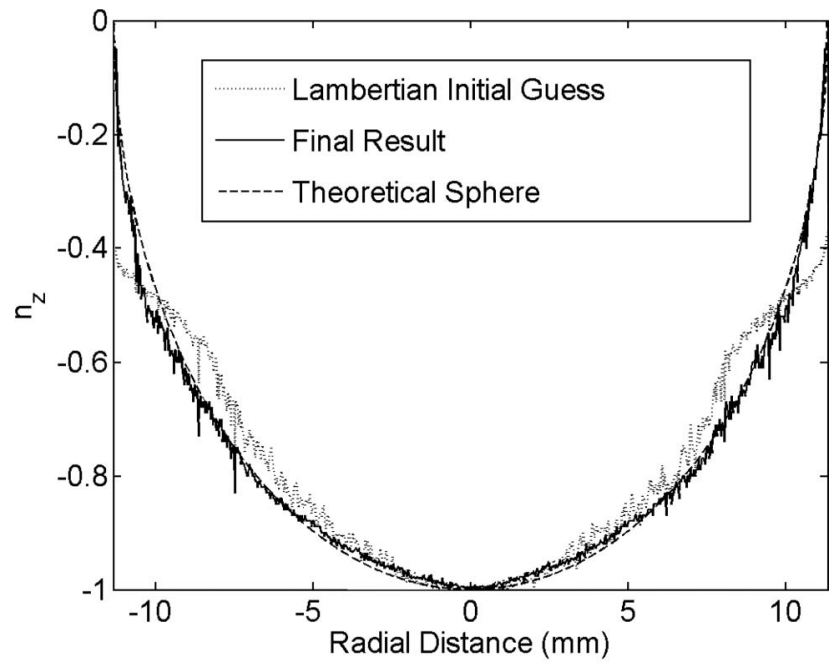

(a)

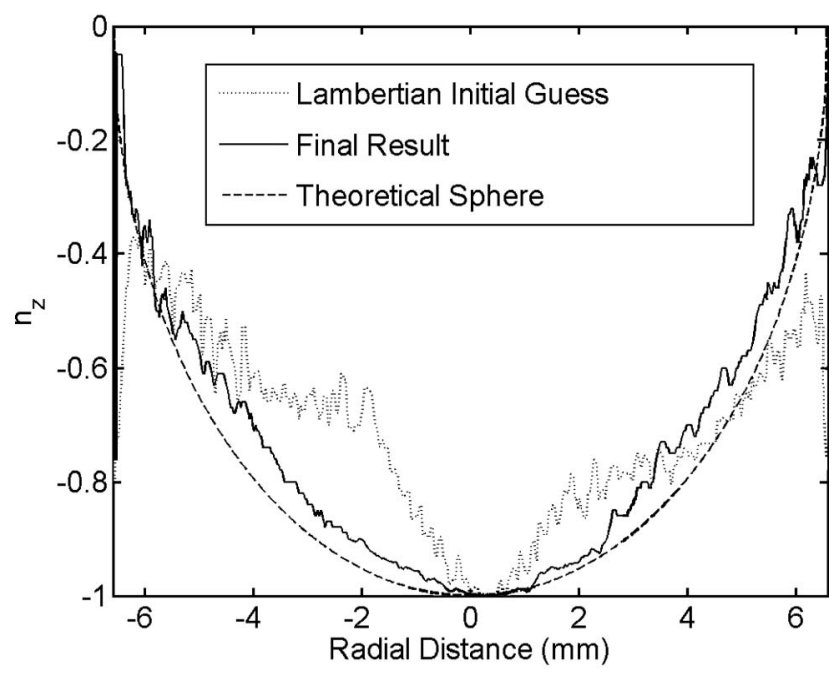

(b)

Fig. 3. $Z$ components of the surface normals of (a) a mousebal and (b) painted ball bearing resulting from photometric stereo analysis. The solid curve represents the initial guess assuming a Lambertian BRDF, the dotted curve the final normal map after BRDF parameter optimization, and the dashed curve the theoretical normal map assuming perfect spheres of the appropriate radii. 


\section{A. Spherical Test Objects}

The absolute accuracy of the scanner (including the processing algorithms) was evaluated by scanning two spherical targets, a $22.7 \mathrm{~mm}$ diameter computer mouseball and a $12.7 \mathrm{~mm}$ diameter ball bearing painted with flat (diffusely reflecting) paint. The former constitutes a Lambertian spherical object, while the reflectance properties of the latter include a diffuse specular term. The data were acquired with the light source at a polar angle of $35.8^{\circ}$ and 12 azimuthal angles spaced at $30^{\circ}$ increments.

Figure 3 shows the results of photometric stereo analysis on the two spheres by comparing the $z$ component of the surface normal of a cut through the center of each sphere to the theoretical values. The calculated normal data shown are from both the initial guess assuming a Lambertian reflectance [Eq. (3)] and the final result [Eq. (5)] after optimization of the BRDF Ward model parameters. As can be seen in Fig. 3(a), the mouseball is much closer to a Lambertian reflector than the painted ball bearing. However, subsequent BRDF and normal optimizations do still make a noticeable improvement to the normal map of the mouseball. The ball bearing [Fig. 3(b)] has a much more significant diffuse specular component to its BRDF. As a result, the initial normal map based on a Lambertian BRDF is grossly misshapen. Again, the optimization of the objective function [Eq. (5)] reveals a much improved set of surface normals.

The resulting parameterized BRDFs of the spheres are compared to purely Lambertian BRDF profiles in Fig. (4). We have chosen to calculate and show the expected reflectance in the view direction over a cut through the center of a spherical target given a par-

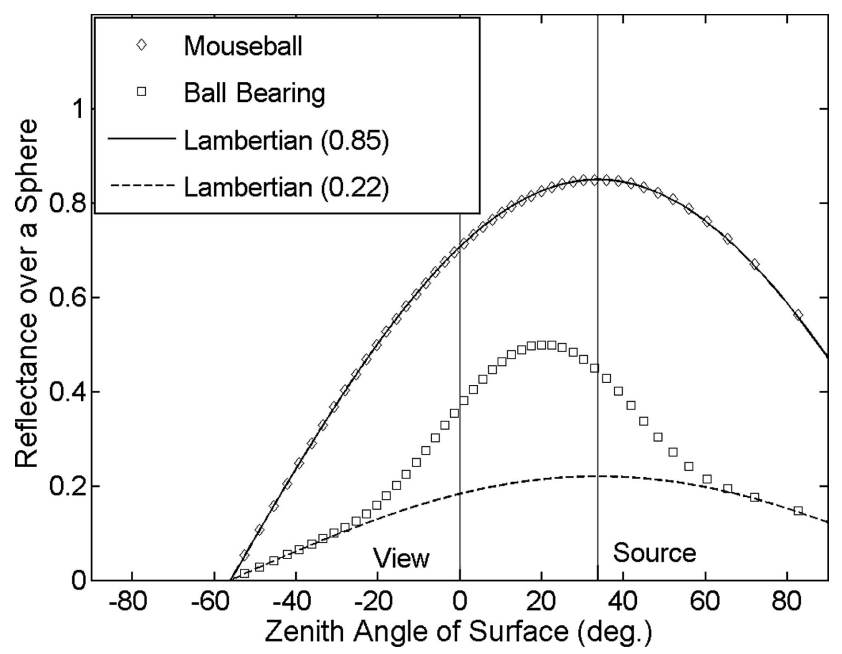

Fig. 4. Reflectance profiles of a cut through the center of a sphere plotted against the zenith angle of the sphere's surface. The view direction is directly overhead the sphere and the incident illumination at a zenith angle of $35.8^{\circ}$. The diamonds represent the reflectance profile given the $\mathrm{BRDF}$ of the mouseball, the squares the BRDF of the painted ball bearing, and the solid and dashed curves Lambertian BRDFs of 0.85 and 0.22 reflectance, respectively. The latter Lambertian BRDF is in fact the Lambertian component of the BRDF of the ball bearing.

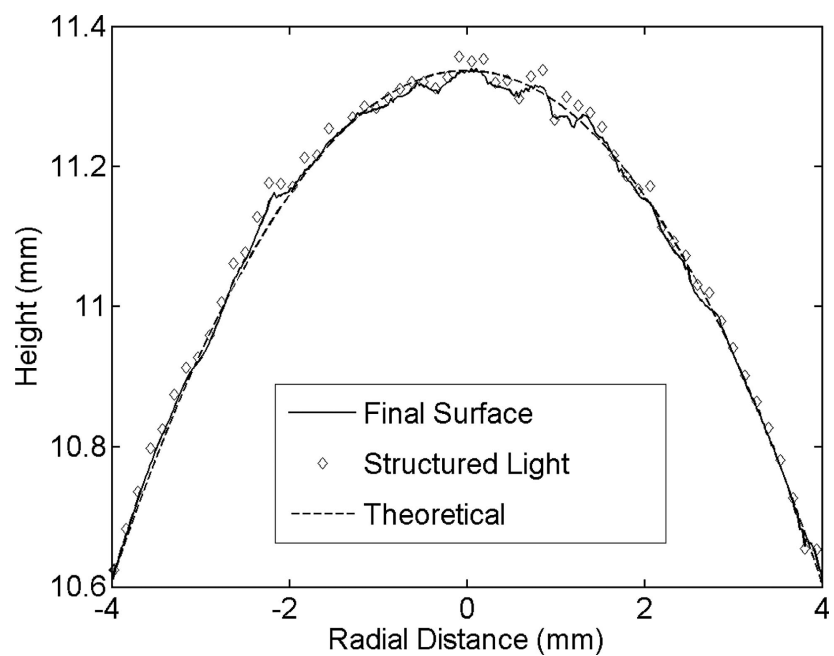

Fig. 5. Height profile of a cut through the center of the mouseball compared to that of a perfect sphere of like radius. The solid curve and diamonds are the final surface height and structured light height of the mouseball (not all points plotted to reduce clutter), respectively, while the dashed curve represents the surface of a perfect sphere.

ticular BRDF and illumination direction. This is directly representative of the observed intensity on the overhead camera with the light source at the polar angle $\left(35.8^{\circ}\right.$. As can be seen in Fig. 4, the BRDF of the mouseball is indistinguishable from that of a Lambertian sphere with a reflectance of 0.85 . The peak reflectance of these BRDFs occurs where the surface normal is oriented directly toward the angle of incident illumination. This is not the case for the painted ball bearing, whose BRDF contains a significant diffuse specular term. The shape of the ball-bearing BRDF is drastically different, and the peak reflectance occurs where the surface normal is between the view and illumination directions, as is expected for a specular reflector.

The height of the mouseball is shown in Fig. 5, which compares the results of structured light anal-

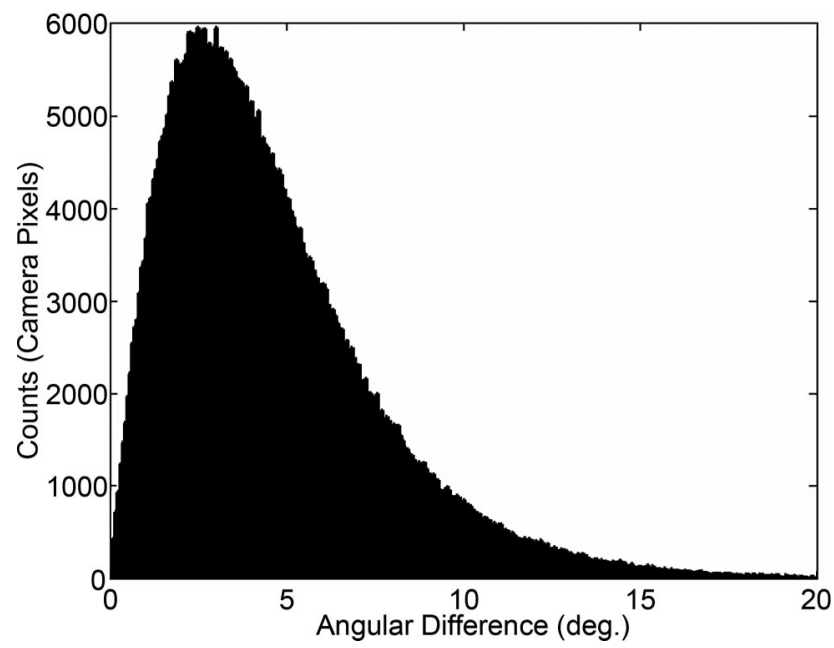

Fig. 6. Histogram of the angular difference between the surface normals of the mouseball and those of a perfect sphere of like radius. 


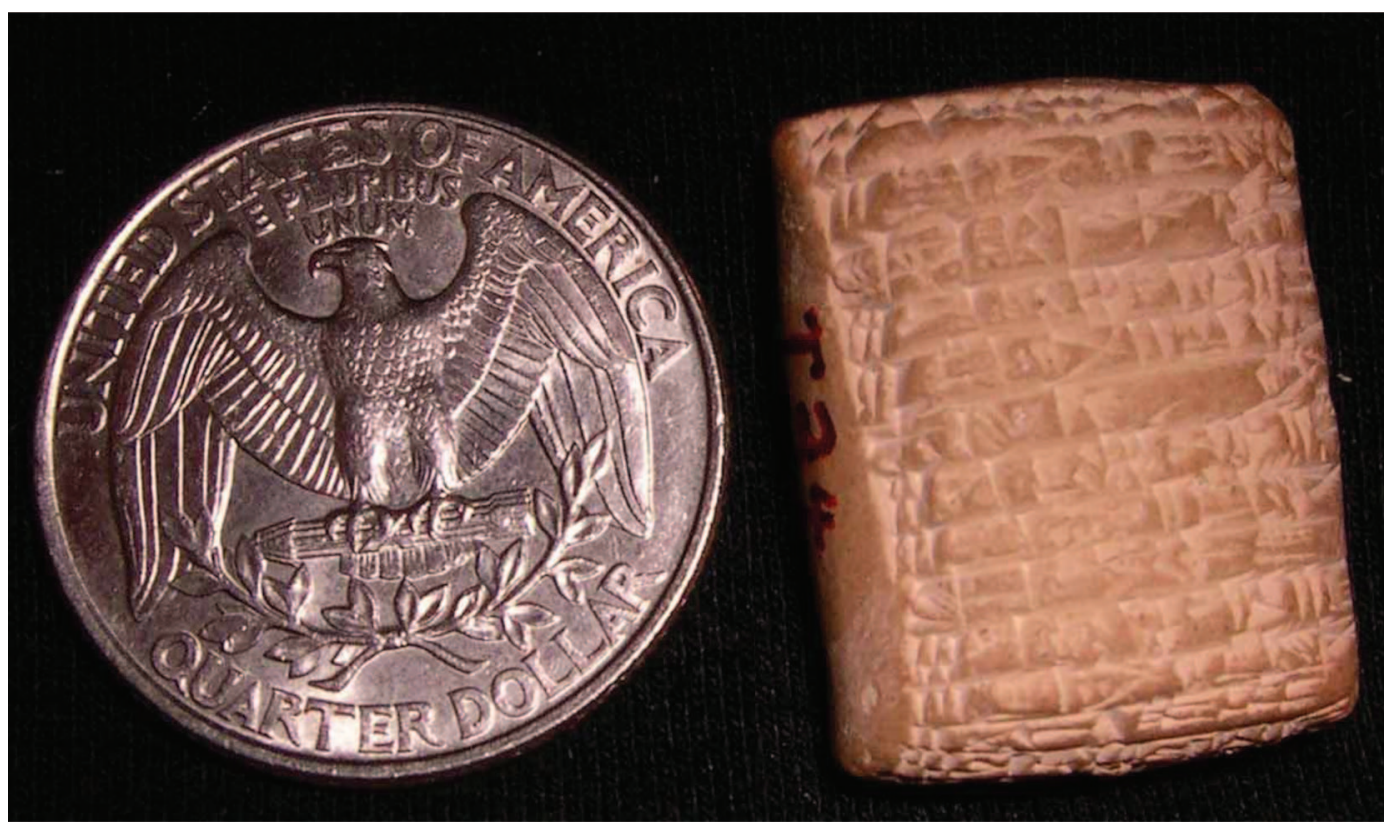

Fig. 7. (Color online) A small cuneiform tablet (test specimen T24) compared to the size of a U.S. quarter.

ysis and the final surface [Eq. (9)] to the theoretical height. We have chosen to show the results of these analyses on the mouseball, the larger of the two spherical targets, to better represent the global resolution of the scanner. By setting the height of a perfect sphere equal to the height of the top of the target, the absolute height deviation was calculated. Over the top half of the mouseball (a distance of over $11 \mathrm{~mm}$ in the vertical direction and $22 \mathrm{~mm}$ in the horizontal direction) $98 \%$ of the height deviation resulting from structured light analysis was within $0.2 \mathrm{~mm}$ with the mean and standard deviations being $67 \mu \mathrm{m}$ (absolute value) and $85 \mu \mathrm{m}$, respectively. These results indicate the excellent global accuracy of the structured light technique. The statistics of the absolute height deviation of the final surface are slightly improved; $98 \%$ of the height deviation was again within $0.2 \mathrm{~mm}$, but the mean and standard deviations were lowered to $59 \mu \mathrm{m}$ (absolute value) and $76 \mu \mathrm{m}$, respectively. The most significant im- provement from the raw structured light result is the reduced noise of the final result, evidence that the minimization algorithm has used the photometric stereo normal data to reduce the noise in the height map. Note that even though the final height looks rough compared with a perfect sphere, the mouseball itself has a rough surface (not perfectly smooth); therefore it is quite plausible that the roughness observed, which is on the order of $50 \mu \mathrm{m}$, is representative of the true surface.

Figure 6 shows a histogram of the angular difference between the final surface normals (resulting from the minimization algorithm) of the mouseball and those of a perfect sphere. The mean and standard deviations of the distribution are $3.3^{\circ}$ and $4.8^{\circ}$, respectively. It is indeterminate how much of this error is attributable to the surface roughness of the mouseball; however, it is evident from Figs. 5 and 6 that both the overall shape and high resolution surface
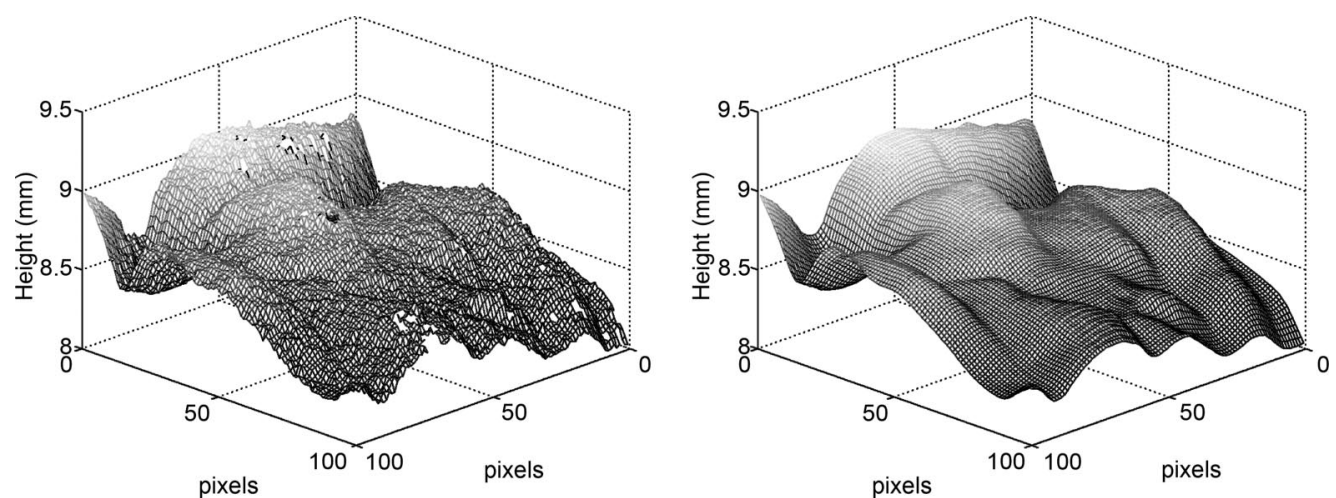

Fig. 8. Meshed surface maps of a $2.68 \mathrm{~mm} \times 2.68 \mathrm{~mm}$ cross section of the T24 tablet showing (left) the structured light height and (right) the final surface. 

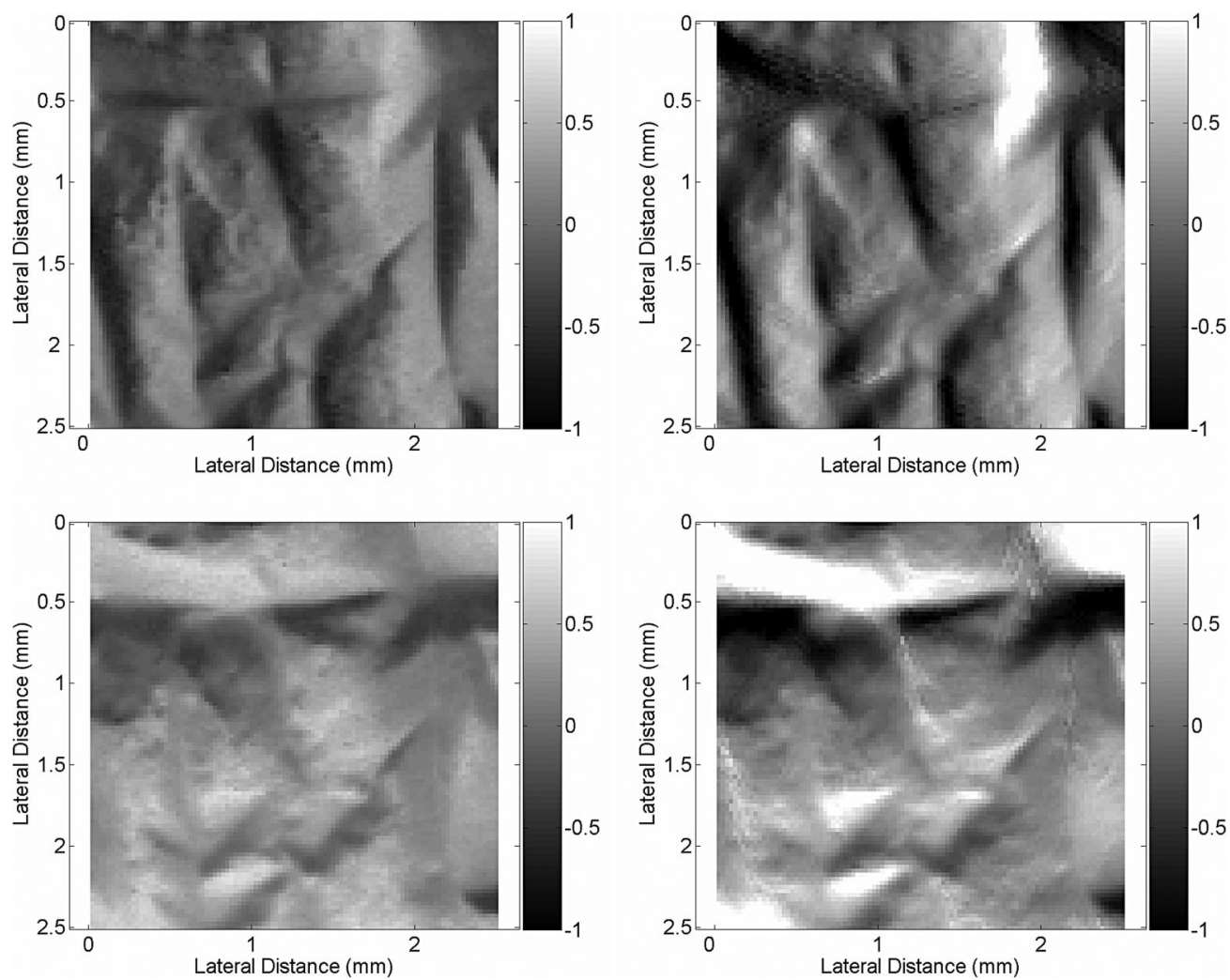

Fig. 9. $x$ (top) and $y$ components (bottom) of the normal vectors over a $2.68 \mathrm{~mm} \times 2.68 \mathrm{~mm}$ cross section of the T24 tablet (left) as measured by the photometric stereo method and (right) computed from the final surface.

orientation of the mouseball are well captured by the scanner.

\section{B. Cuneiform Tablet (T24)}

To put a perspective on the size of the T24 tablet and the resolution of the data, Fig. 7 shows a U.S. quarter next to the T24 tablet. The tablet was scanned using the methods previously described. The meshed surface maps of a $2.68 \mathrm{~mm} \times 2.68 \mathrm{~mm}$ cross section $(100 \times 100$ pixels $)$ of the front of the tablet are shown in Fig. 8; the left mesh shows the structured light height while the right mesh depicts the final surface. These figures substantiate the claim that the minimization algorithm preserves the global height infor- mation resident in the structured light data while discounting the local noise.

The absolute error of this data cannot be calculated without a priori knowledge of the cuneiform surface. To quantify the expected error, an independent experiment consisting of scanning a flat metallic block was performed. The standard deviation of height values over a $2 \mathrm{~mm} \times 2 \mathrm{~mm}$ area of the block were 9 and $2 \mu \mathrm{m}$ for the structured light and final surface height maps, respectively.

Photometric stereo analysis resulted in a $\mathrm{BRDF}$ similar to that of the mouseball-indistinguishable in shape from that of a Lambertian surface, but slightly lower in amplitude due to the reflectivity of
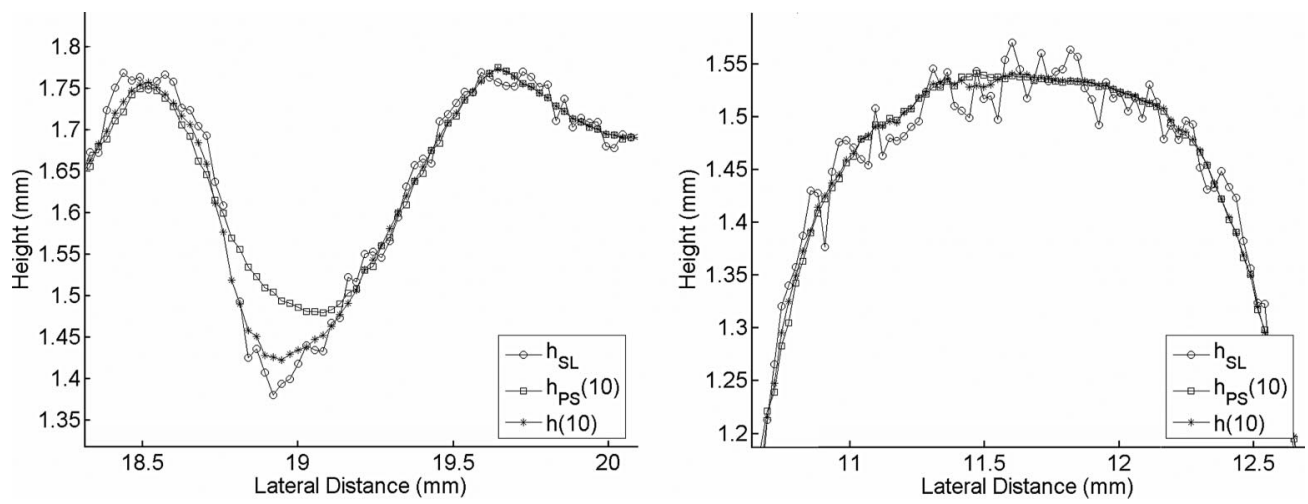

Fig. 10. Height profiles of the T24 tablet. Circles represent the structured light height map. A local integration of the normal data is shown with squares. The stars are the final surface (10 iterations). 


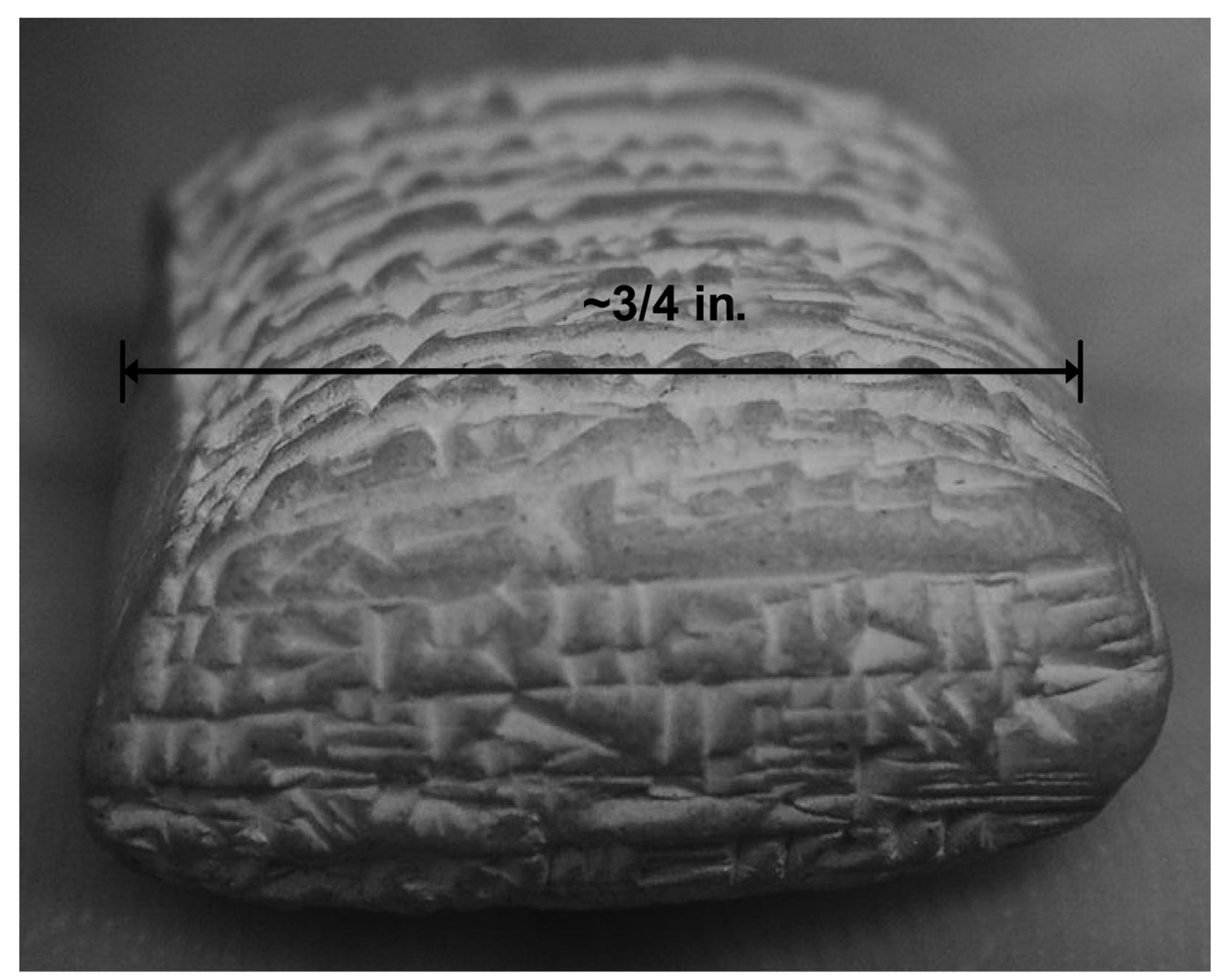

(a)

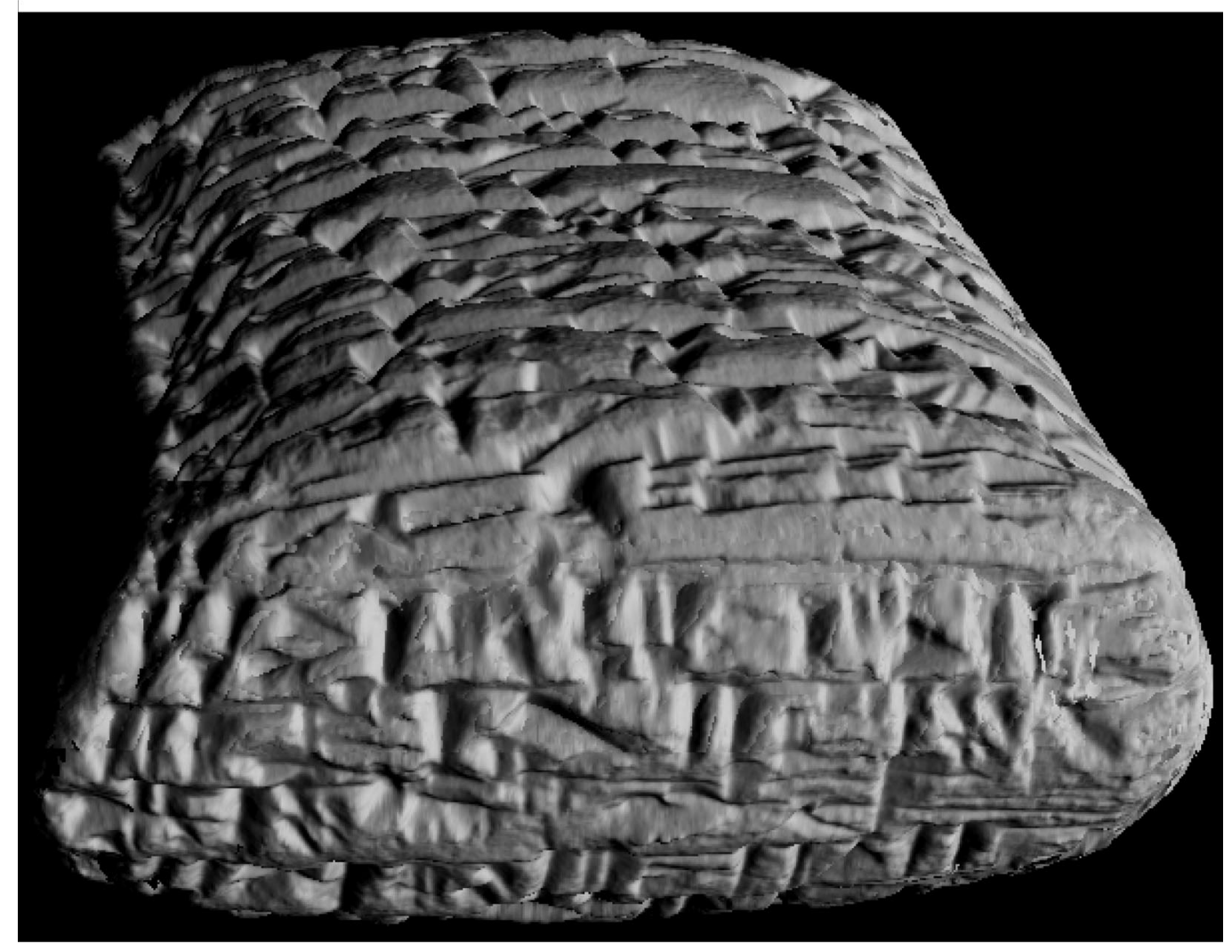

(b)

Fig. 11. (a) Photograph and (b) rendering of the T24 tablet (Ref. 4) from approximately the same view direction. 
the tablet. The $x$ and $y$ components of the photometric stereo normal vectors are shown in the upper plots in Fig. 9 and compared to those calculated from the final surface in the lower plots in Fig. 9. Overall, the slope information resulting from photometric stereo is well preserved in the final surface. In areas of steep slopes, however, the final surface exhibits a slightly steeper slope than the measured normal data. This is because the minimization algorithm adjusts the final surface to more closely match the structured light height in these areas, thereby avoiding an excessive smoothing of the genuine structure.
Example height profiles of tablet data are shown in Fig. 10. Both the integration of the normal map and the final surface suppress the noise of the height data. However, the integration is inaccurate with respect to the genuine structure of the tablet in comparison with the minimization algorithm in areas of steep slopes. This is evident in the center valley in the left plot in Fig. 10. Here, a sharp groove was detected in the structured light data but smoothed over by pure normal integration. The final surface, on the other hand, comes within approximately $50 \mu \mathrm{m}$ of the groove depth as measured by structured light analysis.

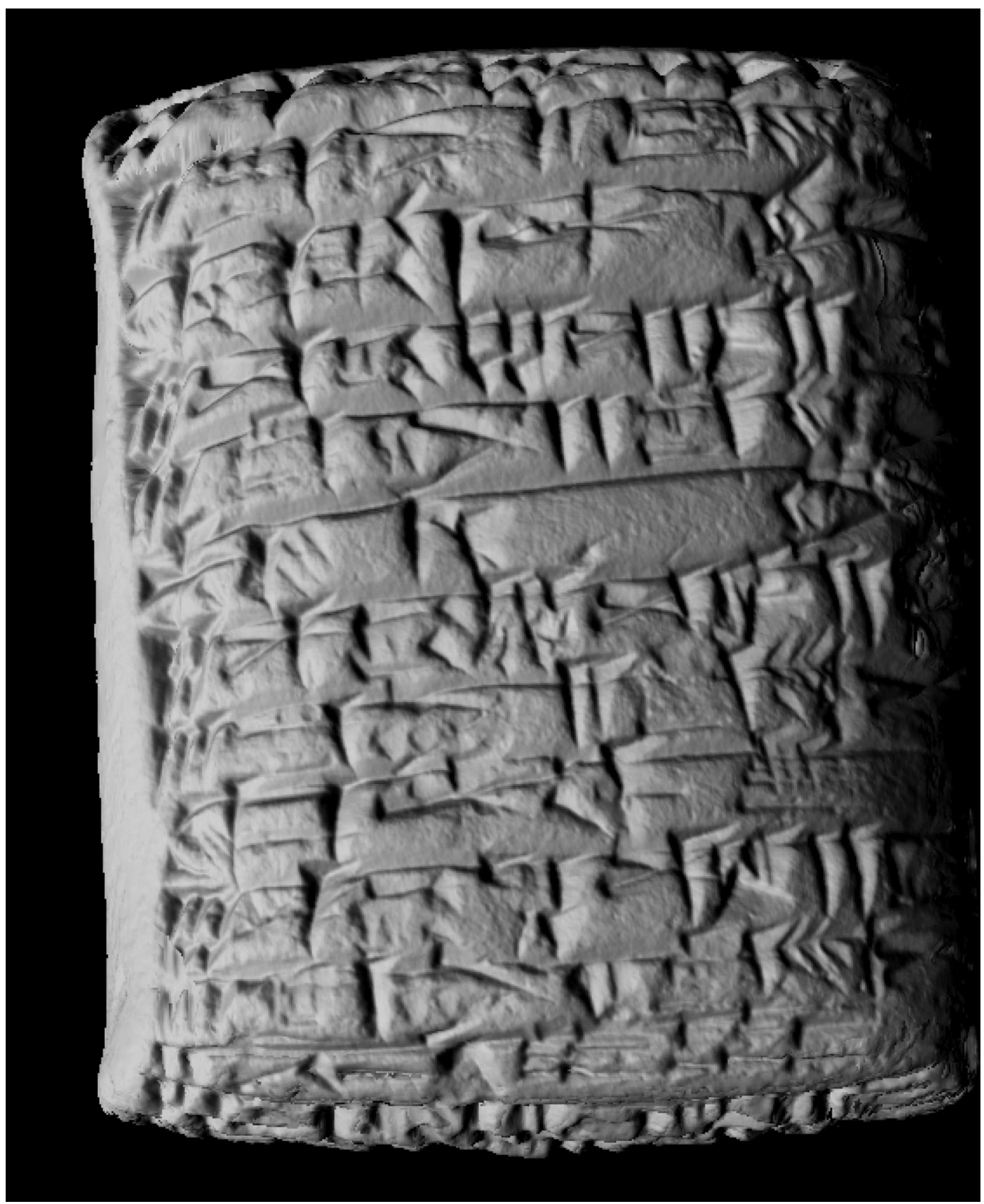

Fig. 12. Rendering of the T24 tablet (Ref. 4) from approximately the same view direction as the photograph in Fig. 7. The distance from the top to the bottom of this rendering is approximately the diameter of a U.S. quarter. 
A photograph of the tablet under ambient lighting is shown in Fig. 11(a), and a rendering from approximately the same view direction and with the light source toward the right is shown in Fig. 11(b). This rendering was created by digitally stitching scans of the tablet front and bottom together. ${ }^{4}$ The position of the light source was chosen to accentuate the features of the tablet in order to demonstrate the utility of having a 3D surface model compared with photographic records. The rotations of both the surface model and the light source to any orientation allow for the best possible rendition of a given tablet feature. The rendering matches the photograph cuneiform character for cuneiform character, wedge for wedge, and also maintains the gross shape of the tablet. This figure pair also points out one of the distinct features of a rendering versus a photograph. Photographs inevitably display a finite depth of field in which some features are sharply in focus and others are blurred. This is not the case for a rendering that inherently has an infinite depth of field. Another rendering is shown in Fig. 12. In this rendering, the view direction matches that of the photograph in Fig. 7.

In addition to photographic comparisons, cuneiform scholars were presented with the T24 tablet and the visualization software portraying the T24 data set. Their observations and comments indicated that all characters were not only clearly and accurately represented, but were observable at a much finer resolution and smaller scale than conventional techniques allow. Other observations and remarks made addressed the accurate reconstruction of dents and surface scratches smaller in scale than cuneiform characters, the ease and safety of visualization, the significant improvement over photographic digitization, and the unprecedented potential for remote study. ${ }^{24,25}$

\section{Conclusion}

We have implemented an incoherent light 3D scanner capable of achieving lateral resolutions ranging from 5 to $100 \mu \mathrm{m}$ or more and depth resolutions of approximately $2 \mu \mathrm{m}$. Associated data-processing algorithms were also detailed; these included photometric stereo techniques that allow for the recovery of the surface normal map and BRDF, a phase-stepping structured light technique, an error minimization algorithm used to combine high resolution normal and low resolution height data, and an object color reconstruction scheme.

The scanner was originally designed to scan cuneiform tablets, and it is for this reason that BRDF determination is of significant importance; the materials and material characteristics of cuneiform tablets vary greatly depending on the time and geographic location of origin. ${ }^{24}$ However, the robust design of the scanner allows for application toward many objects, including but not limited to historical artifacts, fossils, teeth, and bones.

The data presented from spherical objects have shown the high resolution of the scanner on both global and local scales. The scans of a cuneiform tab- let were also presented herein, as well as to scholars in the cuneiform community, who provided complimentary feedback on the data quality and the technology potential. ${ }^{24}$

This work has been supported by the NSF Medium ITR IIS-0205586. The authors thank all those who have collaborated on other aspects of the Digital Hammurabi project, including Subodh Kumar, Dean Snyder, and Lee Watkins. The authors also thank special Jerry Cooper (who was able to arrange for loan of the T24 tablet from the collection at Johns Hopkins University) and Jonathan Cohen and Budirijanto Purnomo (who provided the rendering software with which to view the scanned 3D data) ${ }^{4}$

\section{References}

1. S. Kumar, D. Snyder, D. Duncan, J. Cohen, and J. Cooper, "Digital preservation of ancient cuneiform tablets using 3Dscanning," in Proceedings of IEEE Fourth International Conference on $3 D$ Digital Imaging and Modeling (IEEE, 2003), pp. 326-333.

2. S. E. Anderson and M. Levoy, "Unwrapping and visualizing cuneiform tablets," IEEE Comput. Graphics Appl. 22, 82-88 (2002).

3. S. I. Woolley, N. J. Flowers, T. N. Arvanitis, A. Livingstone, T. R. Davis, and J. Ellison, " $3 \mathrm{D}$ capture, representation, and manipulation of cuneiform tablets," in Three-Dimensional Image Capture and Applications IV, Proc. SPIE 4298, 103-110 (2001).

4. D. V. Hahn, D. D. Duncan, K. C. Baldwin, J. D. Cohen, and B. Purnomo, "Digital Hammurabi: design and development of a 3D scanner for cuneiform tablets," in Three-Dimensional Image Capture and Applications VII, Proc. SPIE 6056, 60560E1-12 (2006).

5. "Cuneiform Digital Library Initiative," http://cdli.ucla.edu.

6. J. Cohen, D. Duncan, D. Snyder, J. Cooper, S. Kumar, D. Hahn, Y. Chen, B. Purnomo, and J. Graettinger, "iClay: digitizing cuneiform," in Proceedings of the Fifth International Symposium on Virtual Reality, Archaeology, and Cultural Heritage (2004), pp. 135-143.

7. N. Demoli, H. Gruber, U. Dahms, and G. Wernicke, "Characterization of the cuneiform signs by the use of a multifunctional optoelectronic device," Appl. Opt. 35, 5811-5820 (1996).

8. F. Dreesen, H. Delere, and G. von Bally, "High-resolution color holography for archaeological and medical applications," in Optics and Lasers in Biomedicine and Culture, Optics within Life Sciences V, C. Fotakis, T. Papazoglou, and C. Kalpouzos, eds. (Springer, 2000), pp. 349-352.

9. Y. Morimoto, T. Nomura, M. Fujigaki, S. Yoneyama, and I. Takahashi, "Deformation measurement by phase-shifting digital holography," Exp. Mech. 45, 65-70 (2005).

10. R. J. Woodham, "Photometric method for determining surface orientation from multiple images," Opt. Eng. 19, 139-144 (1980).

11. J. Pan, P. S. Huang, and F. Chiang, "Color-coded binary fringe projection technique for 3-D shape measurement," Opt. Eng. 44, 23606-1-9 (2005).

12. G. Sansoni, M. Carocci, and R. Rodella, "Three-dimensional vision based on a combination of gray-code and phase-shift light projection: analysis and compensation of the systematic errors," Appl. Opt. 38, 6565-6573 (1999).

13. D. B. Goldman, B. Curless, A. Hertzmann, and S. M. Seitz, "Shape and spatially-varying BRDF's from photometric stereo," in Proceedings of the Tenth IEEE International Conference on Computer Vision (IEEE, 2000), pp. 341-348. 
14. C. Buil, CCD Astronomy (Willmann-Bell, Inc., 1991).

15. G. J. Ward, "Measuring and modeling anisotropic reflection," Comput. Graph. 26, 265-272 (1992).

16. R. Klette and K. Schlüns, "Height data from gradient fields," in Machine Vision Applications, Architectures, and Systems Integration V, Proc. SPIE 2908, pp. 204-215 (1996).

17. W. Tan, Y. Wang, Y. Chen, and B. Xu, "Shape and reflectance recovery based on a photometric stereo approach," in Proceeding 426 Computer Graphics and Imaging, M. H. Hamza, ed. (2004), pp. 350-354.

18. S. Zhang and P. S. Huang, "Novel method for structured light system calibration," Opt. Eng. 45, 083601-1-8 (2006).

19. K. J. Gåsvik, Optical Metrology, 2nd ed. (Wiley, 1995), pp. 254-260.

20. W. H. Press, S. A. Teukolsky, W. T. Vetterling, and B. P. Flannery, Numerical Recipes in $C$, 2nd ed. (Cambridge U. Press, 1996).
21. Q. Kemao, S. Fangjun, and W. Xiaoping, "Determination of the best phase step of the Carré algorithm in phase shifting interferometry," Meas. Sci. Technol. 11, 1220-1223 (2000).

22. D. J. Smith and A. G. Bors, "Height estimation from vector fields of surface normals," in Proceedings of IEEE 14th International Conference on Digital Signal Processing (IEEE, 2002), pp. 1031-1034.

23. J. D. Barchers, D. J. Fried, and J. D. Link, "Evaluation of the performance of Hartman sensors in strong scintillation," Appl. Opt. 41, 1012-1021 (2002).

24. J. Cooper, Professor, Department of Near Eastern Studies, Johns Hopkins University, 3400 N. Charles St., Baltimore, Md. 21218 (personal communication, 2005).

25. D. Snyder, Manager, Digital Hammurabi Project, Computer Science Department, Johns Hopkins University, 3400 N. Charles St., Baltimore, Md. 21218 (personal communication, 2005). 